\title{
ГРАВИТАЦИОННЫЕ ЭФФЕКТЫ
}

DOI: $10.22363 / 2224-7580-2020-4-137-146$

\section{ИСКУССТВЕННЫЙ ЭКСПЕРИМЕНТ ПО УТОЧНЕНИЮ ЗАКОНА ГРАВИТАЦИИ В СОЛНЕЧНОЙ СИСТЕМЕ}

\author{
А.П. Ефремов \\ Институт гравитации и космологии РУДН \\ Российская Федераџия, 115419, Москва, ул. Орджоникидзе, 3
}

\begin{abstract}
Аннотация. Сверхчувствительность гравитационного маневра космического зонда к изменению величины прицельного параметра позволяет организовать искусственный космический эксперимент, целью которого является уточнение закона гравитации в Солнечной системе. Обсуждается упрощенная модель такого эксперимента в космической лаборатории, включающей планетную систему (Солнце, Земля и Венера), в которой зонд (space-ball) движется как пробное тело по баллистической траектории от Земли к Венере (обеспечивающей гравитационный маневр) и обратно к орбите Земли. Мы показываем, что в гравитации Ньютона и Эйнштейна конечные положения зонда (достигаемые в одно и то же время) могут заметно отличаться, что способен фиксировать земной наблюдатель.
\end{abstract}

Ключевые слова: общая теория относительности, космический эксперимент, Солнечная система, космический зонд.

\section{Введение.}

\section{Стоит ли проверять состоятельность гравитации Эйнштейна?}

В своих философских (скорее метафизических) работах мной уже многократно затрагивалась тема научной веры, которая мало отличается от веры религиозной и возникновению которой в наибольшей степени способствует становление научных авторитетов и незыблемость авторитетных мнений. Не так давно на одной из уважаемых конференций я (как докладчик) в прениях задал вопрос аудитории: кто из коллег лично проводил опыты, доказывающие справедливость квантовой механики. Выяснилось - никто. Мы со студенческой скамьи просто верим в то, что эта странная аксиоматическая теория с высокой степенью точности описывает явления микромира (в чем, я, кстати, также не сомневаюсь, но - по другим причинам). Авторитета лекций, 
прочитанных профессорами, и учебников, написанных академиками, оказалось достаточно, чтобы искренне (и навсегда!) поверить в избранность гильбертова пространства, эрмитовость операторов и принцип неопределенности. Притом, что о смысле базового объекта - функции состояния - академики спорят уже почти что век.

Что касается теории гравитации Эйнштейна - общей теории относительности (ОТО), ее идеи, похоже, вполне овладели не только бывшими и нынешними студентами, но и самыми широкими массами. И основная заслуга здесь принадлежит астрономам и экспериментаторам, использующим самую современную технологическую базу - космические наблюдательные системы, мощные радиотелескопы, гигантские интерферометры. В результате последние десятилетия ознаменовались, действительно, значимыми открытиями. В первую очередь это массивные двойные объекты (типа PSRD1913+16, PSR J0737-3039), характеристики которых позволяют подтвердить эйнштейновский характер смещения периастра и - косвенным образом - потерю энергии за счет гравитационного излучения. Наконец - совсем недавнее - прямое (и уже неоднократное) наблюдение всплесков гравитационного поля, очевидно, связанное с прохождением через Солнечную систему гребней гравитационных волн, возникающих при слиянии сверхмассивных двойных объектов. Существенным обстоятельством является тот факт, что эти всплески (в частности первое событие - CW 150914) были замечены сразу на двух интерферометрах, разделенных расстоянием в 3000 км.

Вместе с уже «классическими» наблюдаемыми явлениями - расширения Вселенной и наличия реликтового излучения - эти новые факты, как представляется, еще более укрепляют веру человечества в общую теорию относительности, и эта тема становится все более и более популярной. Сегодня ей уделяют внимание практически все средства передачи информации и серьезные научные журналы, и телевизионные каналы, и многочисленные блогеры-любители, которые переполняют Интернет, разъясняя простому народу детали отличия черных дыр от кротовых нор и темной материи от темной энергии.

На этом фоне как бы меркнут «еще более классические», но скромные свидетельства проявлений ОТО в рамках Солнечной системы - специфическое смещение перигелия планет, гравитационное линзирование звездой Солнце и смещение частоты в красную зону электромагнитного сигнала, выходящего из поля тяготения. Вместе с ними меркнут и прежние сомнения. Однако при этом возникает два вопроса: (1) Так ли уж все очевидно с теорий гравитации Эйнштейна? (2) Не стоит ли прекратить дальнейшие шаги по ее экспериментальной проверке? На оба эти вопроса я бы ответил отрицательно, и вот с какими аргументами.

Да, наблюдения последних лет и их результаты вроде бы выглядят достаточно убедительно. Тем не менее все факты, подтверждающие состоятельность ОТО, по моему мнению, как минимум обладают двумя неприятными свойствами. Во-первых, во всех случаях мы, по сути, имеем дело с единичными наблюдениями очень редких природных явлений - движения и слияния 
двойных массивных объектов. А во-вторых, эти объекты находятся очень далеко от наблюдателя. И если двойные пульсары наблюдаются в нашей Галактике на расстояниях в пределах «всего» 10 тысяч световых лет, то дальность источников впервые детектированного гравитационного излучения определяется примерно в 1,3 миллиарда световых лет. То есть мы имеем дело с событием, отделенным от нас интервалом времени, составляющим около 10 \% времени жизни Вселенной. Так что, имея в виду ненулевую вероятность изменения закона гравитации во времени, вряд ли можно с уверенностью утверждать, что наблюдаемые явления соответствуют сегодняшнему состоянию вещей.

Указанные два обстоятельства, с моей точки зрения, в известной степени подрывают важнейший принцип физического эксперимента: возможность его повторяемости, наличие которой - вместе с повторяемостью результатов - является основой эмпирического установления закона природы, ответственного за наблюдаемое явление. С этих позиций утверждения о безусловной приемлемости теории гравитации Эйнштейна представляются все же недостаточно убедительными.

Однако неконструктивная критика легковесна, и, помимо сомнений, их автору следовало бы высказать некие предложения, которые оказались бы достойны внимания. Контекст вышеуказанных сомнений с определенностью указывает на предложения, содержащие альтернативу предыдущих опытов: надежные эксперименты должны быть непременно повторяемы; лучше всего иметь возможность поставить их специально, и желательно в ближнем космосе - для оценки современных нам закономерностей и экономии времени.

На первый взгляд эта задача практически не решаема: по сути, речь идет о проверке эффектов ОТО в Солнечной системе, где эти эффекты чрезвычайно малы (напомню, что Леверье понадобилось почти полвека наблюдений для выявления аномального смещения перигелия Меркурия). Тем не менее, если бы такую задачу решить удалось, стоит ли игра свеч? Нужно ли еще раз контролировать состоятельность теории гравитации Эйнштейна? Представляется, что в рамках вот такого (почти невозможного) предлагаемого опыта безусловно, да.

Более того, такой эксперимент (точнее серия экспериментов), позволяющий достаточно точно определить закон гравитационного воздействия Солнца, оказывается вполне возможным, он не слишком сложен в реализации и не требует чрезмерных экономических затрат. Но этот космический эксперимент требует точнейших расчетов и высоких технологий - впрочем, вполне отвечающих развитию соответствующих отраслей науки сегодняшнего дня.

\section{1. Гравитационный маневр - навигационная «мода» космических миссий}

Прежде чем перейти к описанию возможного эксперимента по проверке закона гравитации, доминирующего в Солнечной системе, несколько слов о «навигационной моде» в организации космических миссий. 
Конечно, «мода» - термин условный. Тем не менее чуть ли не во всех «экстерриальных» полетах космических аппаратов используется прием так называемого гравитационного маневра (gravity assist maneuver) - ускорение или замедление аппарата в гравитационном поле планеты, вблизи которой намеренно прокладывается его траектория (см. одну из базовых работ [1]). Следует отметить, что впервые этот метод был успешно применен в проекте «Луна 3» в СССР (1993); с тех пор идея и практика такого маневра были реализованы во многих космических проектах, среди них самые известные: Пионер 10 (1972, NASA), Вояджер 1 (1973, NASA), Кассини-Гюйгенс (1997, NASA, ESA, ASI), Розетта (2004, ESA), Джуно (2011, NASA), БепиКоломбо (2018, ESA, JAXA) ${ }^{1}$.

Метод гравитационного маневра сочетает в себе две основные составляющие - непростую математику (анализ задачи трех тел) и высокую технологию (реализация высокоточных траекторий), но решает он, по сути, простую практическую задачу - возможность существенной экономии химического (или иного вида) топлива, необходимого для надлежащего ускорения космического аппарата, направленного к назначенной цели. Столь прозаическая (казалось бы) причина приверженности «моде» гравитационного маневра имела своим следствием как минимум два существенных обстоятельства.

Первое - и в теории и на практике выяснилось, что успех такого маневра в высшей степени зависит от точности вычисления (а затем и практического воплощения) так называемого прицельного параметра космического аппарата при его приближении к планете, отдающей часть своей (для ускорения) или отбирающей у аппарата (для замедления) некоторую энергию. Математически прицельный параметр есть кратчайшее расстояние между планетой и линией относительной скорости «апnарат - планета» (на условной бесконечности); совсем упрощенно этот параметр можно ассоциировать с минимальным расстоянием между аппаратом и планетой ${ }^{2}$. Так вот на практике выяснилось, что это минимальное расстояние должно быть выдержано с точностью до десятков метров, то есть примерных размеров самого аппарата [2]. И это на фоне расстояний, которые аппарат преодолевает при движении по околосолнечной орбите - а они сопоставимы с примерным расстоянием от Земли до Солнца - 150 млн км. Таким образом, относительная точность соблюдения величины прицельного параметра оказывается феноменальной: $\sim 10^{-10}$.

Второе непосредственно следует из первого - в расчетах движения космического аппарата приходится учитывать влияние всех возможных физических факторов, существенных для удовлетворения указанной точности. Это и гравитационное воздействие других планет и оказавшихся поблизости астероидов, и несферичность Солнца, и - не исключено - снос аппарата солнечным ветром. Но среди этих факторов неожиданно оказалась и необходимость

\footnotetext{
${ }^{1}$ NASA - National Astronautic and Space Administration (USA), ESA - European Space Agency, JAXA - Japan Aerospace Exploration Agency.

${ }^{2} \mathrm{~B}$ реальном гравитационном маневре минимальное расстояние «аппарат - планета» называется «перицентр»; это расстояние всегда несколько меньше прицельного параметра. 
учитывать отклонение закона гравитации от его классической ньютоновской формы $^{3}$. Детали расчетов космических миссий по понятным причинам не афишируются, но, как можно судить, в каждом отдельном случае разработчики таких проектов стараются принять во внимание также и эффекты ОТО.

Изучение десятков открытых работ (хорошая библиография в [3]) по теме гравитационного маневра (ГМ) продемонстрировало отсутствие системного изучения функции «чувствительности» такого маневра к величине прицельного параметра (ПП), и эта тема стала предметом внимания последних нескольких лет моей небольшой исследовательской группы. Основные (и удивительные!) результаты этих исследований частично опубликованы в [4; 5], о них - в следующем разделе.

\section{2. Гравитационный маневр - мощный усилитель малых физических эффектов}

Базой анализа чувствительности ГМ к изменению ПП послужила виртуальная модель идеализированной планетной системы, включающей центральную звезду и две планеты, на одной из которых осуществляется запуск космического зонда и находится наблюдатель, оценивающий чувствительность маневра, реализуемого второй планетой (рис. 1).

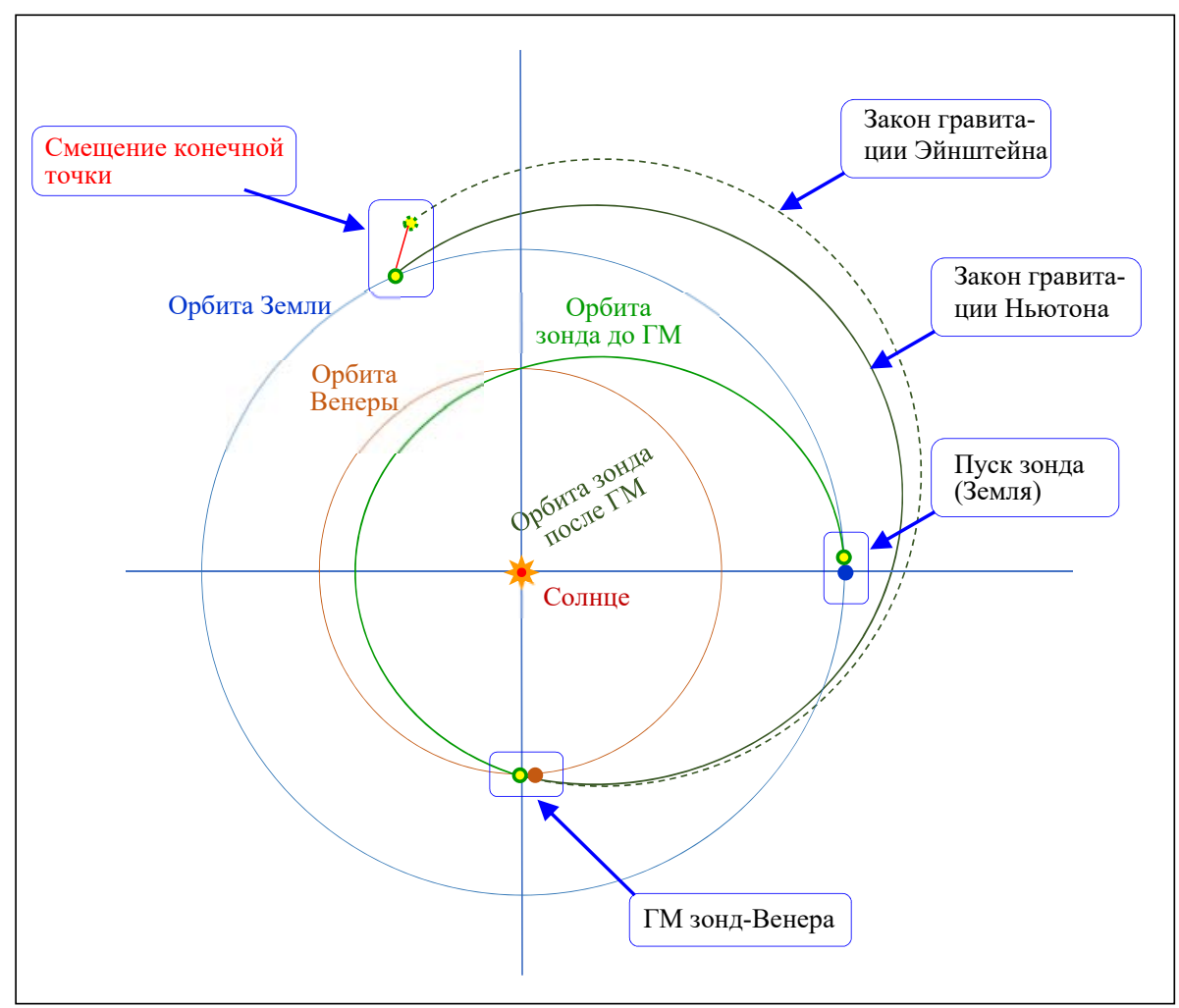

Рис. 1. Схема полета зонда: Земля (пуск) - Венера (ГМ) - конечная точка

\footnotetext{
${ }^{3}$ На это указал один из рецензентов моей статьи, написанной в соавторстве и посвященной оценке чувствительности гравитационного маневра к величине прицельного параметра в гравитации Ньютона.
} 
Несложно понять, что данная схема имитирует Солнце, Землю и Венеру, но для простоты считается, что планеты движутся по круговым орбитам (с постоянной линейной скоростью). Однако остальные физические параметры тел вполне соответствуют наблюдательным данным. Для определения функции чувствительности достаточно, чтобы в планетной системе действовал закон гравитации Ньютона. В этих условиях сначала предлагается осуществить следующий математический (мыслительный) эксперимент [4].

С земной орбиты к орбите Венеры по баллистическим траекториям падают два космических зонда так, что в установленное время они осуществляют ГМ со сравнительно небольшими, но слегка различными прицельными параметрами (например, 18000 и 17999 км, то есть разность ПП зондов 1 км). Здесь зонды ускоряются и по новым баллистическим траекториям возвращаются к орбите Земли, где одновременно подают сигнал, позволяющий наблюдателю достаточно точно определить их положение в пространстве. Очевидно, в конечный момент времени зонды окажутся в различных точках пространства; наблюдаемое расстояние между ними определяет значение функции чувствительности ГМ при данном значении ПП ${ }^{4}$. Последовательно выбирая ряд характерных значений ПП зондов от 18 до 8 тыс. км (с фиксированной разностью в 1 км), мы, таким образом, получаем набор значений чувствительности ГМ и можем построить эмпирический график соответствующей функции.

Однако обладая необходимым набором физических данных (массы, начальные координаты и скорости тел, форма закона гравитации), мы можем математически - с хорошей степенью точности - вычислить все значения функции чувствительности ГМ в рамках рассматриваемой модели. И это можно сделать как минимум двумя способами.

Первый способ - детально проработанная поколениями математиков схема описания ГМ с помощью приближенного решения задачи трех тел методом «конических отрезков» - "Patched Conic Approximation" (PCA). В этом варианте зона гравитационного влияния планеты (зона Хилла) на фоне размеров околосолнечной траектории зонда считается почти точечной, время собственно ГМ - практически мгновенным, а траектория зонда в поле планеты описывается единственной гиперболой. В методе PSA цепочка сравнительно простых уравнений имеет своим результатом график теоретически подсчитанных значений функции чувствительности ГМ (рис. 2, $a$ ).

Второй способ - прямое вычисление [5] положения зондов в каждый момент времени (с интервалом в 1 с) по известным формулам классической динамики с использованием специального приложения программной системы Python 3. Достаточно точное построение траекторий каждого зонда требует расчета до 50 млн точек, при этом весь полет зондов занимает примерно 1,5-2 года и разность конечных положений пары зондов автоматически дает

\footnotetext{
${ }^{4}$ Строго говоря, в данной модели эксперимента функция чувствительности ГМ определяется как отношение разности конечных положений зондов к разности значений прицельного параметра (которая в данном случае принята равной единице).
} 
значение чувствительности ГМ как функции величины прицельного параметра (рис. 2, б).

$a$

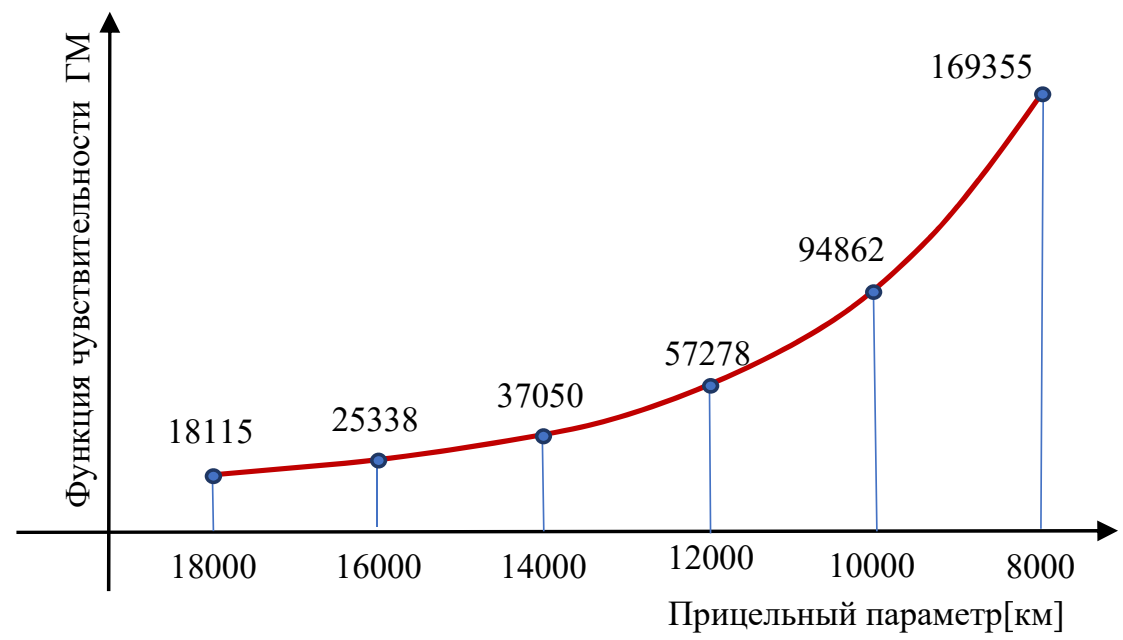

$\sigma$

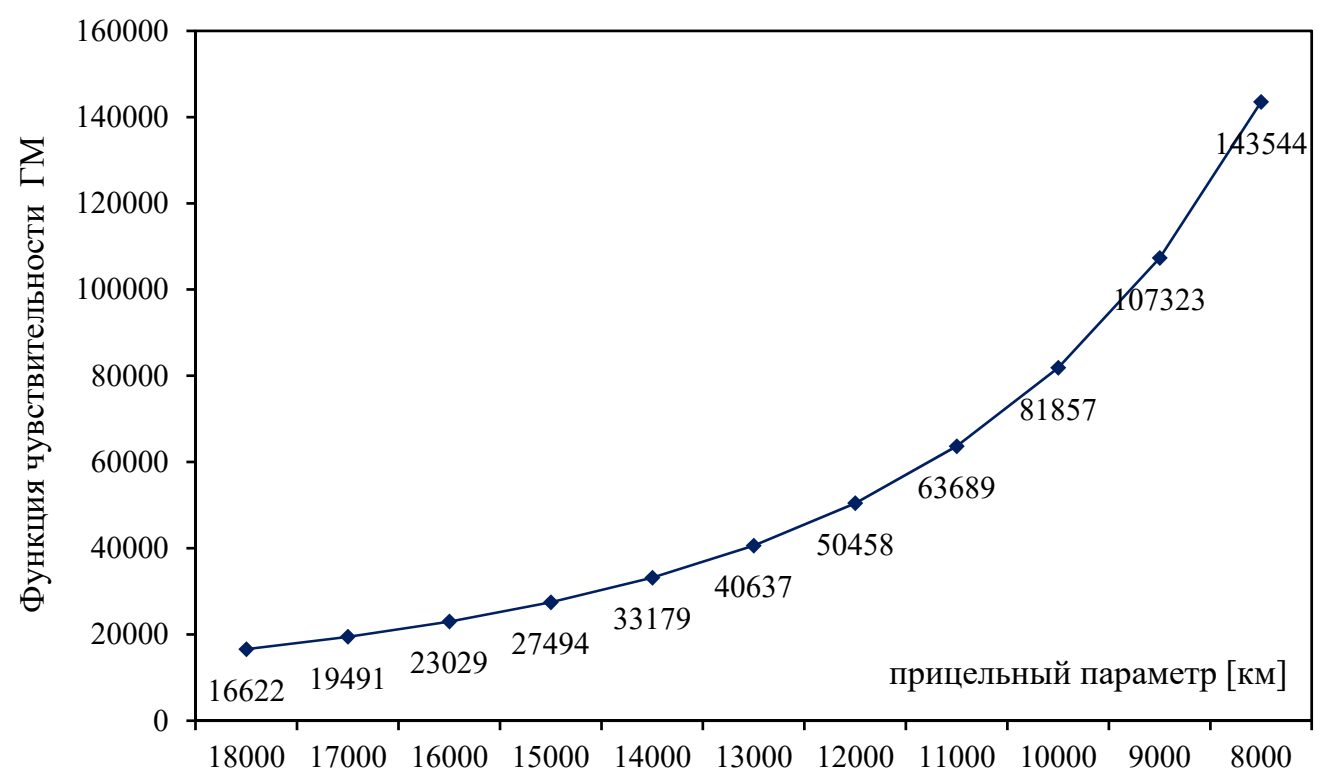

Рис. 2. Математический расчет функции чувствительности ГМ (a); численный расчет функции чувствительности ГМ (б)

Анализ графиков функции чувствительности ГМ на рис. $2, a-\sigma$, полученных двумя различными способами, позволяет сделать следующие выводы.

(i) Оба способа дают не вполне совпадающие, но близкие значения функции; для страховки будем ориентироваться на меньшие значения (рис. 2, б).

(ii) Но и в этом случае чувствительность ГМ по отношению к изменению значения ПП оказывается чрезвычайно высокой: при ПП 18000 км отклонение на 1 км дает в конечной точке смещение на 17000 км, тогда как при ПП 9000 км (всего 3 тыс. км над поверхностью Венеры) то же отклонение в 1 км дает смещение в конечной точке более чем на 100000 км. Таким образом, ГМ 
можно условно рассматривать как мощнейший «усилитель» физических эффектов, вызывающих малые отклонения космических тел на их околосолнечных орбитах, в том числе изменение расчетного прицельного параметра предстоящего гравитационного маневра. Это небольшое изменение непременно проявится как очень существенное отклонение от запланированного положения в финальной точке орбиты.

Это свойство сверхчувствительности ГМ, по-видимому, можно использовать для уточнения формы закона гравитации, действующей в нашей планетной системе. Имеется в виду, конечно, поле тяготения Солнца - самого существенного гравитационного агента. Для планет Солнечной системы, по крайней мере первых четырех, имеющих сравнительно малую массу, различия между законами гравитации Ньютона и Эйнштейна практически нет, поэтому для определения чувствительности ГА мы использовали классический закон тяготения.

\section{3. Расчет и реализация эксперимента по уточнению закона гравитации}

В гравитации Эйнштейна основные параметры движения космического зонда, направляемого по баллистической траектории от Земли к Венере в гравитационном поле Солнца, теоретически определяются как интегралы энергии и момента импульса, следующие из уравнения геодезической для метрики Шварцшильда [6]. Этих величин достаточно, чтобы по известным классическим формулам с удовлетворительной точностью вычислить значения эксцентриситета и большой полуоси квазиэллиптической орбиты зонда, присущей эйнштейновскому закону гравитации. При этом путь зонда от точки пуска до встречи с Венерой оказывается немного короче за счет небольшого «сжатия» орбиты и поворота ее перигелия, так что факт ГМ происходит немного раньше, чем при тех же начальных условиях это имело бы место в гравитации Ньютона. Это означает, что несколько меньше оказывается и величина прицельного параметра. Математический расчет по перечисленным формулам показывает, что ПП (и перицентр - минимальное расстояние между зондом и планетой) в теории Эйнштейна уменьшается примерно на 30 км. Тогда, согласно графику функции чувствительности, изображенному на рис. 2, б, в заданный момент времени (снова у орбиты Земли) зонд может отклониться от «ньютоновской» конечной точки более чем на миллион километров.

Для проверки правильности теоретических вычислений мы провели контрольный численный расчет траектории зонда в эйнштейновской гравитации по точкам, каждая из которых соответствовала последующей секунде полета (используя, как и раньше, приложение Python 3). Основной задачей в этом блоке вычислений оказалась правильная запись формы гравитационной силы, подставляемая в правую часть уравнения динамики. Здесь выяснилось, что возможен единственный вариант такой записи силы - в касательном 
пространстве метрики Шварцшильда; только в этом случае модуль силы гравитации ОТО оказывается чуть больше, чем модуль силы гравитации Ньютона. И тогда квазиэллипс орбиты зонда оказывается чуть меньшего размера и поворачивается в «нужную сторону» в полном соответствии с известной формулой смещения перигелия. При этом конечная точка траектории зонда также может смещаться в сторону более чем на миллион километров (в зависимости от величины прицельного параметра).

Итак, для проведения эксперимента все расчеты движения зонда целесообразно планировать в гравитации Ньютона, с достаточной точностью определив величину прицельного параметра (пожалуй, не менее 14 тыс. км) и координаты конечной точки (на орбите Земли). Затем - также с надлежащей точностью - осуществляется пуск зонда по баллистической траектории в сторону Венеры, после чего эксперимент до самого конца продолжается «в автоматическом режиме». Зонд подлетает к Венере, осуществляет гравитационный маневр, в результате которого ускоряется и вновь подходит к орбите Земли, где в установленный момент времени посылает сигнал, фиксирующий конечную точку. Если наблюдатель обнаруживает зонд вблизи заданных координат (в рамках заранее рассчитанной погрешности), то это означает, что закон гравитации скорее близок к ньютоновскому. Существенное же отклонение зонда от заданной точки (значительно больше расчетной погрешности) должно свидетельствовать о справедливости закона гравитации Эйнштейна.

Все предварительные расчеты такого эксперимента, конечно, нужно проводить машинными методами (как, собственно, оно и делается на практике). Не исключено, что эта кропотливая работа, предполагающая учет всех физических факторов и многократный контроль, может оказаться наиболее затратной, поскольку технологические системы выглядят не слишком сложными: это носитель (ускоритель), позволяющий достаточно точно задать начальные данные движения зонда, и сам зонд - симметричное тело (шар) массой 300-500 кг, который по возможности минимально реагирует на все взаимодействия, за исключением гравитационных, и способен подавать электромагнитные сигналы. Чтобы не засорять космос, в конечной точке своего полета этот space-ball мог бы просто взорваться, превратившись в свет и газ.

Значительных препятствий для осуществления и повторения эксперимента такого рода, как представляется, нет. Тем более что его уже сегодня могут реализовать известные государственные и международные агентства, а также частные компании. Успешности такой работы будет способствовать и непременная исследовательская конкуренция. Без сомнения, возникнут и новые научные содружества. В результате - всего за несколько лет - может накопиться определенная статистика, которая позволит с хорошей степенью достоверности уточнить закон гравитации, действующий в Солнечной системе, и сверх того - вполне возможно попутно способствовать экспериментальному открытию новых физических эффектов.

Хочется верить, что это окажется еще один шагом к познанию устройства мира. А когда мы узнаем, как устроен мир, мы поймем зачем в нем мы. 


\title{
Литература
}

1. Егоров В.A. О некоторых задачах динамики полета к Луне // УФН. 1957. LXIII-1. 73-117.

2. Jehn R. BepiColombo "Mercury Cornerstone Consolidated Report on Mission Analysis"// MAS Working Paper No. 525. 26 January, 2015. Issue 5.2. BC-ESC-RP-05500, ESOC, Robert-BoschStr. 5, 64293 Darmstadt, Germany.

3. Ceriotti M. Global optimization of multiple gravity assist trajectories. University of Glasgow, Dpt. Aerospace Engineering, 2010.

4. Yefremov A.P. Sensitivity of the Gravity Assist to Variations of the Impact Parameter // Gravit. and Cosmol. 2020. 26 (2). P. 118-123.

5. Yefremov A.P., Vorobieva A.A. A Planet's Gravity Assist as a Powerful Amplifier of Small Physical Effects in the Solar System, submitted to Acta Astronautica, 2020.

6. Yefremov A.P., Vorobieva A.A. A "Space-Ball" Experiment in the Solar System May Clarify the Nature of Gravity, ready to submission in 2020.

\section{AN ARTIFICIAL EXPERIMENT AIMED TO SPECIFY THE GRAVITY LAW IN THE SOLAR SYSTEM}

\author{
A.P. Yefremov \\ Institute of Gravitation and Cosmology of RUDN University \\ 3 Ordzhonikidze St., Moscow, 115419, Russian Federation
}

\begin{abstract}
Ultra-sensitivity of a planet's gravity assist (GA) to changes of the test-body impact parameter prompts a space experiment testing the nature of gravitational field in the Solar system. The Sun, Earth and Venus serve as the space lab with a primitive space probe (ball) as a test body moving on a ballistic trajectory from the Earth to Venus (rendering GA) and backwards to the Earth's orbit. We show that in Newton and Einstein gravity, the probe's final positions (reached at the same time) may differ greatly; an Earth's observer can measure the gap.
\end{abstract}

Keywords: general theory of relativity, space experiment, solar system, space probe. 